Methods Data from waves 1 (2009-2011, $\mathrm{n}=6051), 2$ (2012, $\mathrm{n}=5487)$ and $3(2014-2015, \mathrm{n}=4623)$ of the Irish Longitudinal Study of Ageing (TILDA), a stratified probability sample prospective cohort, was analysed. Frequency of participation in seven social activities ('Go to films, plays, concerts', 'Attend classes or lectures', 'Travel for pleasure', 'Play cards, bingo or games', 'Go to pub', 'Eat out of house' and 'Participate in sport or exercise') was collected. Depressive symptoms were assessed using the 8-item Centre for Epidemiological Studies Depression (CESD) scale, and chronic disease count included self-reported doctors' diagnosis of cardiovascular (heart attack, angina, stroke, transient ischaemic attack, heart failure) and non-cardiovascular chronic conditions (high blood pressure, diabetes, arthritis, lung disease, osteoporosis). Multilevel mixed effects logistic regression modelling was employed to assess the effect of changes in 1) chronic disease count and 2) depressive symptoms on each item of social participation (defined as monthly participation or less) over three waves, adjusted for socio-demographic and health covariates.

Results Mean age at baseline was $63.2 \mathrm{y}$ and $46.9 \%$ of the sample were male. Rates of social participation remained stable across waves. Each additional chronic disease accrued was associated with decreased participation in 'Attend classes or lectures' (Odds Ratio (OR): 0.86, 95\% CI: 0.74-0.99) and 'Participate in sport or exercise' (OR: 0.86, 95\% CI: 0.770.97 ) and an increase in 'Go to pub' (OR: 1.28, 95\% CI: 1.09-1.50). A one unit increase in depressive symptoms over time was associated with decreased participation in 'Participate in sport or exercise' (OR: 0.96, 95\% CI: 0.93-0.99) only.

Conclusion This longitudinal analysis suggests that deterioration of physical and mental health may influence specific domains of social participation in community dwelling older adults. Holistic approaches to disease management and mental health interventions in older age should include programmes to facilitate and maintain social and leisure time activities.

\section{OP72 RELIGIOUS ATTENDANCE, LONELINESS AND DEPRESSIVE SYMPTOMS IN MIDDLE AGED AND OLDER WOMEN IN IRELAND}

J Orr*, K Tobin, RA Kenny, C McGarrigle. The Irish Longitudinal Study on Ageing, Trinity College Dublin, Dublin, Ireland

\subsection{6/jech-2017-SSMAbstracts.71}

Background Evidence for an association between mental health and religiosity largely supports a positive effect of religiosity on mental health. However, there remains a lack of research into the underlying mechanisms of these associations involving other social and health factors, particularly in older women. We aimed to investigate causal pathways between religious attendance and depressive symptoms, and test whether this relationship is mediated by loneliness.

Methods We analysed three waves of The Irish Longitudinal Study on Ageing (TILDA) (2000011, 2012, 2014-2015), a stratified probability cohort of men and women aged over 50 resident in Ireland. A total of 3400 women were included in this analysis. A theoretical longitudinal model of religious attendance and depressive symptoms was tested using Structural Equation Modelling (SEM), adjusted for age, marital status, self-rated health, education and recent adverse life events. Log likelihood tests were used to compare model fit. Depressive symptoms were measured using the 8-item Centre for Epidemiologic Studies Depression Scale (CES-
D) and loneliness with the UCLA Loneliness Scale. Religious practice and beliefs were also collected. Changes in religious attendance between waves were calculated. All analyses were conducted using Stata 14.

Results A majority of women attended religious services (86\%) and $60 \%$ attended at least once a week at baseline, with a decrease in attendance at subsequent time points $(85 \%$ and $57 \%$ at Wave $2 ; 84 \%$ and $55 \%$ at Wave 3). Mean (SD) depressive symptoms were 3.38 (4.07) at Wave $1 ; 3.19$ (3.99) at Wave 2; and $3.62(4.03)$ at Wave 3. Regular attendance at Wave 1 and Wave 2 predicted fewer depressive symptoms at Wave 2 (Incident Rate Ratio (IRR):0.81 95\% CI:0.73-0.89) and Wave 3 (IRR:0.92 95\% CI:0.86-1.00) respectively. When loneliness was included in the model, the effect of regular attendance remained unchanged between Wave 1 and Wave 2 . The effect was attenuated, but not mediated, between Wave 2 and Wave 3 (IRR: 0.94 95\% CI: 0.87-1.02). Depressive symptoms consistently predicted changes in attendance, with higher depressive symptoms predicting subsequent increased and decreased attendance.

Conclusion Longitudinal analyses of religious attendance and depressive symptoms suggest this is a complex relationship which is at least in part bidirectional. Evidence did not support loneliness as a mediator of this relationship. Analyses using other measures of religiosity may help further elucidate these associations.

\section{Policy analysis}

\section{OP73 SYSTEMS SCIENCE FOR CARIBBEAN HEALTH: THE DEVELOPMENT OF A SYSTEM DYNAMICS MODEL FOR GUIDING POLICY ON DIABETES IN A RESOURCE LIMITED SETTING}

${ }^{1} \mathrm{~L}$ Guariguata*, ${ }^{2,3} \mathrm{C}$ Guell, ${ }^{1} \mathrm{TA}$ Samuels, ${ }^{4} \mathrm{EAJA}$ Rouwette, ${ }^{2} \mathrm{~J}$ Woodcock, ${ }^{1} \mathrm{IR}$ Hambleton, ${ }^{1,2} \mathrm{~N}$ Unwin. ${ }^{1}$ Faculty of Medical Sciences, The University of the West Indies, Bridgetown, Barbados; ${ }^{2}$ MRC Epidemiology Unit, University of Cambridge, Cambridge, UK; ${ }^{3}$ European Centre for Environment and Human Health, University of Exeter, Truro, UK; ${ }^{4}$ Nijmegen School of Management, Radboud University, Nijmegen, The Netherlands; ${ }^{5} \mathrm{MRC}$ Epidemiology Unit, University of Cambridge, Cambridge, UK

\subsection{6/jech-2017-sSMAbstracts.72}

Background Type 2 diabetes (T2DM) is a major cause of morbidity and mortality in the Caribbean and a threat to development. Halting its rise by 2025, a WHO target, requires interventions addressing the determinants of unhealthy diet and physical inactivity. The objective of this project is to engage with stakeholders in the development of a system dynamics (SD) simulation model on the effect of different policy interventions on diabetes prevalence and mortality in the Caribbean.

Methods Following SD methodology, we used a mixed methods approach to combine interviews $(n=13)$ with stakeholders from multiple sectors across four countries (Barbados, Belize, Jamaica, and Saint Vincent and the Grenadines) with existing data on regional NCD policy and quantitative evidence. An additional twenty-two interviews from stakeholders in 7 Caribbean countries that were conducted as part of an ongoing policy evaluation study were also reviewed. Participants were sampled from existing contacts and their referrals. Analysis was guided by iterative thematic analysis using a grounded approach. 
A subgroup of stakeholders participated in a group modelbuilding workshop to develop causal maps on the determinants of physical inactivity, unhealthy diet and effective healthcare delivery in the Caribbean. Data from the qualitative and quantitative reviews were applied to further develop the causal structures.

Results Stakeholders reinforced the need for upstream interventions including the provision of a healthy food environment and sustainable urban development facilitating active living, supported by fiscal incentives (subsidies) and disincentives (taxes). Stakeholders emphasised car-based development as a major driver of physical inactivity including a lack of infrastructure for active transport, a high social regard for car usage and a lack of social support for physical activity. Unhealthy diet, according to stakeholders, was driven by changing social structures in the home, the marketing and distribution of highly processed foods, and limited access to locally produced fresh fruits and vegetables. The causal maps are serving as the basis for a stakeholder-driven policy simulation model that will be validated against quantitative evidence. Conclusion The interconnectedness of diabetes determinants highlights the importance of a multi-sectoral response and a systemic approach to decreasing the diabetes burden. The simulation model aims to aid Caribbean policymakers in evaluating the potential impact of different policy options on future diabetes prevalence and mortality. The overall utility of this approach to stakeholder engagement and option appraisal will be assessed and developed further for use in other resourceconstrained settings.

\section{OP74 UNDERSTANDING THE PROCESS OF DEVELOPING AND IMPLEMENTING CHRONIC DISEASE POLICIES IN THE CARIBBEAN REGION: A QUALITATIVE POLICY ANALYSIS}

${ }^{1,2} \mathrm{C}$ Guell ${ }^{*},{ }^{3} \mathrm{MM}$ Murphy, ${ }^{3,4} \mathrm{TA}$ Samuels, ${ }^{3} \mathrm{~L}$ Bishop, ${ }^{2,4} \mathrm{~N}$ Unwin. ${ }^{1}$ European Centre for Environment and Human Health, University of Exeter, Truro, UK; ${ }^{2} \mathrm{MRC}$ Epidemiology Unit and Centre for Diet and Activity Research (CEDAR), University of Cambridge, Cambridge, UK; ${ }^{3}$ Faculty of Medical Sciences, University of the West Indies, Cave Hill Campus, Barbados; ${ }^{4}$ Chronic Disease Research Centre, University of the West Indies, Bridgetown, Barbados

\subsection{6/jech-2017-SSMAbstracts.73}

Background The Caribbean, like other middle income regions, experiences a significant burden of non-communicable diseases (NCDs), and responded in 2007 with a heads of government declaration committing to a range of policy measures. In 2015, this multi-method qualitative evaluation study investigated the progress made in seven Caribbean countries; this paper reports the findings of an analysis of the process that helped or hindered the development and implementation of policies.

Methods We conducted analysis of relevant policy documents, and 76 semi-structured interviews with 80 stakeholders from government (ministries of health, education); civil society (academia, agricultural, health and church organisations) and private businesses. Interviews were conducted by two regional teams. The initial pragmatic coding and categorisation of data with the software Dedoose was deductively framed by the WHO NDC Action Plan, and guided by a Multiple Streams policy approach and realist principles. This was complemented by further inductive thematic analysis and synthesis by the authors.
Results Two overarching themes emerged related to the process that guided the development, adaptation and implementation of regional policy goals into local settings.First, stakeholders reported a commonly shared recognition of the need for multi-sectoral partnerships but were frustrated by the difficulties of achieving this in practice. Country-specific NCD commissions served as case studies for both positive experiences of achieving 'whole of society' collaboration and difficulties in equitable representation and translation into government-led action. In settings with more effective multisectoral working it was possible to identify 'policy entrepreneurs' who had the profile and traction to promote cross sectoral action. Second, stakeholders highlighted the importance and opportunity provided by policy transfer. When comparing policy creation across small islands, most success has been made in areas such as tobacco control that could use an international framework and roadmap as a template. As policy efforts were most advanced in larger, more resource-rich countries, some stakeholders called for more synergetic working on policy solutions across countries. They felt that policy transfer could be improved in a region of relatively small countries but benefitting from a strong regional agenda, cooperation and professional exchange.

Conclusion Stakeholders' local and regional insight, expertise and experience helped to explain the processes that supported or hindered effective policy-making in different settings or different policy areas. Triangulating their reflections with a document review helped to corroborate and strengthen their assessment, provide contextual information to reports and also identified gaps in documentation and narratives.

\section{OP75 CATALYSING GLOBESITY: AN ANALYSIS OF THE IMPACT OF US FREE TRADE AGREEMENTS ON CALORIC INTAKE FROM A NATURAL EXPERIMENT IN CANADA USING THE SYNTHETIC CONTROL METHOD}

${ }^{1} \mathrm{P}$ Barlow*, ${ }^{2} \mathrm{M}$ McKee, ${ }^{1} \mathrm{D}$ Stuckler. ${ }^{1}$ Department of Sociology, University of Oxford, Oxford, UK; ${ }^{2}$ Department of Health Services Research and Policy, London School of Hygiene and Tropical Medicine, London, UK

\subsection{6/jech-2017-SSMAbstracts.74}

Background Free trade and investment agreements (FTAs) are often implicated in the global pandemics of overweight, obesity, and related diseases. Concerns centre on how FTAs increase population exposure to unhealthy, high calorie diets rich in salt, sugar, and fat. Yet the empirical evidence to support these concerns is limited, both methodologically and substantively. Thus, few existing studies of FTAs and dietary change have accounted for unobserved confounding, and many were unable to differentiate the impact of FTAs from contemporaneous macroeconomic and policy changes. Moreover, few have examined any potential effect of FTAs on total caloric intake, despite its critical role in the aetiology of obesity. Here we address these limitations by analysing a unique natural experiment from the unanticipated implementation of the Canada-US Free Trade Agreement (CUSFTA) in 1989.

Methods We use data from the UN Food and Agricultural Organisation and the synthetic control method to test the hypothesis that CUSFTA increased caloric intake in Canada. We also anlayse whether these changes were accompanied by increased US investment and imports in the Canadian food and beverage sector. Finally, we simulate the population impact on weight gain of these changes in caloric intake using 